\author{
Lanqing Zhao ${ }^{1}$, Chunna Zhao', \\ Zhongdong $\mathrm{Du}^{2}$ and Hongwu $\mathrm{Du}^{{ }^{*}}$ \\ ${ }^{1} 112$ Lab, School of Chemistry and Biological \\ Engineering, University of Science and Technology \\ Beijing, Beijing, China \\ ${ }^{2}$ Department of Pediatrics, Beijing Children's \\ Hospital Affiliated to Capital Medical University, \\ Beijing, China
}

Dates: Received: 18 January, 2016; Accepted: 10 February, 2016; Published: 20 February, 2016

*Corresponding author: Hongwu Du, PhD, School of Chemistry and Biological Engineering, University of Science and Technology Beijing, 30 Xueyuan Road, Beijing 100083, P. R. China, Tel: +86 10 62334497; Fax: +8610 62332462; E-mail: hwdu93@126.com

www.peertechz.com

ISSN: 2455-2976

Keywords: Lactate dehydrogenase; Kawasak disease; Expression and purification; Antigenicity analysis; Autoimmune target

\author{
Research Article
}

\section{Lactate Dehydrogenase is involved in but not the Target Antigen in Children with Kawasaki Disease}

\begin{abstract}
Background: Kawasaki disease (KD) is an acute vasculitis often complicating coronary arterial lesions, and gradually becomes the leading cause of acquired heart disease instead of rheumatism. Previous studies often regards elevation of serum lactate dehydrogenase (LDH) level as an auxiliary diagnosis marker when judging myocardial diseases.

Methods: We analyzed the serum LDH levels in newly confirmed KD patients, compared with febrile non-KD patients and healthy donors as controls. Then a subtype of LDH protein (LDHA) were over-expressed and purified for antigenicity analysis of potential anti-LDHA autoantibodies in IgA, IgM and IgG isotype by homemade ELISA, respectively.

Results: An obvious elevation serum levels of LDH was found in $5 / 27$ KD patients $(19 \%, p<0.05)$ and $16 / 27$ febrile non-KD patients $(59 \%, p<0.001)$. None of IgA or IgM isotype of anti-LDHA antibodies were detected in all cases, and only low titer IgG antibodies were detected in KD patients $(7 \%)$ and febrile non-KD patients $(11 \%)$

Conclusions: Our results demonstrated the LDH was associated but not the main autoimmune target in children with $\mathrm{KD}$.
\end{abstract}

\section{Abbreviations}

AP: Acute Pneumonia; BP: Bronchopneumonia; DTT: Dithiothreitol; FC: Febrile Control; HC: Healthy Control; IVIG: Intravenous Immunoglobulin; KD: Kawasaki Disease; LDH: Lactate Dehydrogenase; LDHA: Lactate Dehydrogenase A; MIP: Multiple Infection Pneumonia; MP: Mycoplasma Pneumonia; MS: Mass Spectrometry; VP: Viral Pneumonia;

\section{Introduction}

Kawasaki disease (KD), mainly affects children younger than five years old, is an acute vasculitis first described in 1967 [1]. Although KD remains a mysterious disease and has still not been fully understood $[2,3]$, infection was generally thought playing an important role in the KD pathogenesis [3]. Some microorganism, especially bacteria and viruses, may be the first "driver" of KD [4], but no specific pathogen and other autoimmune target have been precisely identified [2-5]. The diagnosis of $\mathrm{KD}$ is still based on clinical characteristics, and generally fever plus five specific criteria are required for diagnosis of $\mathrm{KD}$ according the suggestion by the American Heart Association [3,6]. So far, no laboratory serum test could auxiliary diagnose KD with specificity $[6,7]$. Thus, some sick children who delayed diagnosis and treatment of KD may lead to severe coronary arterial lesions or even death. Currently, about $25 \%$ KD patients without timely confirmed diagnosis will further develop coronary artery aneurysms [8]. To date, KD gradually becomes one of the leading causes of acquired heart disease worldwide for infants and young kids [9-13].

Lactate dehydrogenase (LDH) is extensively existed in many human somatic tissues and organs. Serum level of LDH is widely considered as an auxiliary marker in early stage of diseases to predict progression or severity of tissue damage in various diseases including necrotising enterocolitis, nasopharyngeal carcinoma and pneumonia [14-17]. For KD patients, LDH level > $590 \mathrm{IU} / \mathrm{L}$ is proposed as one predictor for assessing resistance to intravenous immunoglobulin (IVIG) therapy [18,19]. Macrophage activation syndrome, a complication of $\mathrm{KD}$, is also characterized by elevated serum $\mathrm{LDH}$ $[20,21] . \mathrm{LDH}$ are homotetramers or hereotetramers composed of muscle and heart subunits, which is encoded by LDHA and LDHB gene. More specially, LDHA is made up of four muscle subunits and expressed in most somatic tissues.

In this study, elevation of $\mathrm{LDH}$ was found in some KD patients and febrile non-KD patients. To investigate the potential correlation between KD and LDHA, recombinant human LDHA was overexpressed in E. coli Rosetta (DE3) and purified by Ni-chelating affinity chromatography. Then anti-LDHA autoantibodies were tested for IgA, IgM, IgG isotype by homemade ELISA with sera from confirmed KD patients, febrile non-KD patients and healthy donors, and correlation between serum LDH level and anti-LDHA antibodies were analyzed.

\section{Materials and Methods}

\section{Samples}

All serum samples were provided from Beijing Children's Hospital Affiliated to Capital Medical University during March of 2013 to Jan of 2014. Totally 71 subjects were enrolled in this study, including 27 KD patients, 27 febrile non-KD and 17 age matched healthy donors, and detailed clinical data were listed in Supplementary Table 1. The 
criteria of $\mathrm{KD}$ diagnosis was according to the 5 th revised edition issued by the Japan Kawasaki Disease Research Committee in 2002 [22-24]. Febrile non-KD patients were all confirmed pneumonia patients with more than 3 days fever, and fall into 5 categories: mycoplasma pneumonia, viral pneumonia, acute pneumonia, bronchopneumonia and multiple infection pneumonia. Serum LDH levels were analyzed by UniCel DxC 600 Synchron Clinical Systems (Beckman Coulter Inc, CA). The research conformed to the Declaration of Helsinki Principles.

\section{Expression and purification}

The procedure of human LDHA expression and purification was performed by following our routine method [25]. Briefly, LDHA gene was amplified by PCR with template cDNA from EA.hy926 cells. After digested by endonucleases, target gene was inserted into pET-28a-c(+) vectors by T4 DNA ligase, and then transformed into E. coli Rosetta (DE3) (CW biotech, Beijing, China). Next, the recombinant E. coli was cultured in solid LB medium with added $50 \mu \mathrm{g} / \mathrm{mL}$ kanamycin at 37 , overnight. Then several colonies were picked visually and proliferated separately in fresh $5 \mathrm{~mL}$ liquid LB medium added with $50 \mu \mathrm{g} / \mathrm{mL}$ kanamycin at 37, $200 \mathrm{rpm}$. After OD reached 0.4 0.6, IPTG was added with final concentration of $1 \mathrm{mM}$. Five hours later, the E. coli cells were acquired by centrifugation at 4 , $12000 \mathrm{rpm}$ and ultrasonically disrupted on ice. Then the supernatant and insoluble fractions were analyzed by SDS-PAGE, respectively. Finally, the recombinant LDHA was purified by Ni-chelating affinity chromatography, followed by desalination and renaturation.

\section{Mass spectrometry}

MS analyses were applied to identify results of expression of LDHA [26]. Firstly, the positive protein band on SDS-PAGE gel was excised manually and transferred to a clean $1.5 \mathrm{~mL}$ tube. Then the gel was cut into $1-2 \mathrm{~mm}^{3}$ cubes, then washed with $100 \mu \mathrm{L} 25 \mathrm{mM}$ $\mathrm{NH}_{4} \mathrm{HCO}_{3}$ in $50 \%$ acetonitrile for $15 \mathrm{~min}$. After drying in a vacuum centrifuge (Savant Instruments, NY), the pieces were added with $100 \mu \mathrm{L} 10 \mathrm{mM}$ dithiothreitol (DTT) in $25 \mathrm{mM} \mathrm{NH}_{4} \mathrm{HCO}_{3}$, then incubated for $2 \mathrm{~h}$ at 37 . Next, solution of $55 \mathrm{mM}$ iodoacetamide in $25 \mathrm{mM}^{\mathrm{NH} 4 \mathrm{HCO}^{3}}$ took place of the DTT by same volume, and incubated at room temperature for $1 \mathrm{~h}$ in the dark. Afterwards, the pieces were washed with $100 \mu \mathrm{L} 50 \%$ acet onitrile in $25 \mathrm{mM} \mathrm{NH}_{4} \mathrm{HCO}_{3}$ for 10 min. After dried by the vacuum centrifuge, the gel was added $20 \mu \mathrm{L} 50$ $\mathrm{mM} \mathrm{NH}_{4} \mathrm{HCO}_{3}$ buffer containing $12.5 \mathrm{ng} / \mu \mathrm{L}$ trypsin (Sigma-Aldrich, $\mathrm{MO}$ ) and then incubated for $1 \mathrm{~h}$ at 4 . Then the supernatant was removed and replaced by same volume $50 \mathrm{mM} \mathrm{NH}_{4} \mathrm{HCO}_{3}$ without trypsin to continue digesting for $12 \sim 16 \mathrm{~h}$ at 37 . Finally, the pieces were applied to MS analysis by LC-MALDI-TOF/TOF (ABI45800 Proteomics Analyzer, Applied Biosystem, MA).

\section{Clinical validation}

The ELISAs were performed according our standard procedure with slight modifications [26-28]. In brief, recombinant LDHA was diluted at $100 \mathrm{ng} / \mathrm{mL}$ by dissolving in $0.05 \mathrm{M}$ carbonic buffer and then added into 96 -well microplate $100 \mu \mathrm{L}$ per well. The plates were placed quietly overnight at 4 and then blocked by $5 \%$ goat serum for $2 \mathrm{~h}$ at 37. Next, sera of KD patients, febrile non-KD patients and healthy controls (diluted at 1:1000) were respectively added and incubated for $1 \mathrm{~h}$ at 37 . Then the 96-well plates were washed by $1 \%$ PBST buffer, followed by incubating HRP-conjugated goat anti-human IgA, IgG, and IgM antibody (diluted at 1:10000) for $1 \mathrm{~h}$ at 37, respectively. After that, an ELISA reader (Tecan, Switzerland) was used for quantitative data analysis at $450 / 620 \mathrm{~nm}$.

\section{Statistical analysis}

Peptide mass fingerprints were analyzed by Mascot (Matrix Sciences, UK), and protein scores greater than 70 were considered significant $(p<0.05)$. Data of LDH serum levels and ELISA were analyzed by SPSS 17.0 (SPSS Inc, IL), and differences were evaluated by $\mathrm{t}$-test. If $\mathrm{p}$ value were $<0.05$, statistical significance should be considered. The cut-off value of positive definition was defined as Mean +3 SD.

\section{Results}

\section{Clinical significance of sera LDH levels}

Sera LDH levels were respectively measured in $\mathrm{KD}$ and controls (Table 1). LDH levels were 247 (178-301) IU/L in healthy children, and the cut-off value was defined as $348 \mathrm{IU} / \mathrm{L}$. Figure 1 showed significant differences in $\mathrm{KD}(\mathrm{p}=0.0352)$ and febrile non-KD ( $\mathrm{p}=0.0005)$, with respective LDH level of 294 (152-573) IU/L and 440 (233-1058) IU/L. Elevation of LDH were observed in 5/27 (19\%) KD patients and 16/27 (59\%) febrile non-KD. The results proved lactate dehydrogenase not only increasing in KD patients, but with a higher elevation in other conditions.

\section{Expression and purification of recombinant LDHA}

The procedure generally divided into plasmid construction,

Table 1: Clinical characteristics in children with Kawasaki disease and febrile controls.

\begin{tabular}{|c|c|c|c|c|c|c|}
\hline \multicolumn{2}{|l|}{ Diagnosis } & \multirow{2}{*}{$\begin{array}{c}\text { Median age (range) } \\
2.56(0.25-9.75)\end{array}$} & \multirow{2}{*}{$\begin{array}{c}\text { Male (\%) } \\
16(59)\end{array}$} & \multirow{2}{*}{$\begin{array}{c}\text { LDH (U/L) } \\
294(152-573)\end{array}$} & \multirow{2}{*}{$\begin{array}{c}\text { Positive rate } \\
19 \%(5 / 27)\end{array}$} & \multirow{2}{*}{$\begin{array}{l}\text { P value } \\
0.0352^{*}\end{array}$} \\
\hline $\mathrm{KD}(\mathrm{n}=27)$ & & & & & & \\
\hline$F C(n=27)$ & $\begin{array}{l}\operatorname{MP}(n=9) \\
\operatorname{VP}(n=5) \\
\operatorname{AP}(n=3) \\
\operatorname{BP}(n=5) \\
\operatorname{MIP}(n=5)\end{array}$ & $\begin{array}{l}4.57(1.33-6.75) \\
1.58(0.75-2.67) \\
0.56(0.25-0.92) \\
2.10(1.00-3.67) \\
2.82(1.50-4.92)\end{array}$ & $\begin{array}{l}5(56) \\
2(40) \\
2(67) \\
4(80) \\
4(80)\end{array}$ & $\begin{array}{c}391(276-693) \\
436(233-912) \\
417(310-626) \\
373(245-434) \\
611(314-1058)\end{array}$ & $\begin{array}{l}67 \%(6 / 9) \\
40 \%(2 / 5) \\
33 \%(1 / 3) \\
80 \%(4 / 5) \\
60 \%(3 / 5)\end{array}$ & $\begin{array}{c}0.0001^{* * *} \\
0.0073^{* *} \\
0.0009^{* * *} \\
<0.0001^{* * *} \\
0.0001^{* * *}\end{array}$ \\
\hline $\mathrm{HC}(\mathrm{n}=17)$ & & $3.26(1.42-8.50)$ & $15(88)$ & $247(178-301)$ & $0 \%(0 / 17)$ & - \\
\hline
\end{tabular}

*The cut-off value of positive definition was defined as Mean + 3SD.

*KD, Kawasaki disease; FC, febrile controls; MP, mycoplasma pneumonia; VP, viral pneumonia; AP, acute pneumonia; BP, bronchopneumonia; MIP, multiple infection pneumonia; HC, heathy controls. 
expression and purification. LDHA gene was amplified by PCR and electrophoresed in agarose gel as shown in Figure 2A, corresponding to LDHA theoretical length (999 bp). The information of sense primer: 5'-CCGGAATTCCGGATGGCAACTCTAAAGGATC-3', and the anti-sense primer: 5'-CCCAAGCTTGGGTTATTATGCACTCTTCTTCAAACG-3'. The target DNA were cut by Eco Rand Hind, to form sticky ends to insert into same digested pET28 plasmid, followed by transformation into competent E. Coli, as shown in Figure 2B.

After recombinant E. Coli cultured in solid LB medium, four visual colonies were picked and continued to culture and induce by IPTG. Then whole cell protein were respectively electrophoresed in SDS-PAGE gel (Lane 2-5 in Figure 2C), compared with IPTG control (Lane 6), blank plasmid (Lane 7) and blank E. coli control (Lane 1). Obvious protein bands were found between $35 \mathrm{kD}$ and $40 \mathrm{kD}$ in Lane 2- Lane 5, while other groups didn't.

After ultrasonication, the supernatant and precipitation were respectively loaded for SDS-PAGE analysis, and the results proved LDHA insoluble (Figure 2D). LDHA protein was purified by Nichelating affinity chromatography, followed by desalination and renaturation (Figure 2E). The Recombinant protein was identified by LC-MALDI-TOF/TOF, proved to be LDHA (Figure $2 \mathrm{~F}$ and Supplementary Table 2).

\section{Prevalence of serum anti-LDHA antibodies in KD}

To test the potential antigenicity of LDHA, the recombinant protein was coated on 96-well plates and analyzed for serum IgA, IgG and IgM antibodies, respectively. An elevation of sera anti-LDHA IgA antibody levels were observed in $\mathrm{KD}$ patients compared to febrile non-KD ( $\mathrm{p}=0.035$, Figure $3 \mathrm{~A}$ ), with average OD of 0.503 vs 0.358 . Reactivity of serum anti-LDHA IgA antibodies were detected in none of $\mathrm{KD}$ or febrile non-KD patients. For sera anti-LDHA IgG antibody analysis, some difference was found between KD patients and HC ( $p=0.001$, Figure $3 B$ ), with average OD of 0.465 vs 0.393 . Serum antiLDHA IgG antibodies were detected in 2 of $27 \mathrm{KD}$ patients (7\%), 3 of 27 non-KD patients (11\%) and none in healthy donors. On the other hand, serum anti-LDHA IgM antibodies were detected decreasing in $\mathrm{KD}$ patients compared with disease $(\mathrm{p}=0.020)$ and healthy controls

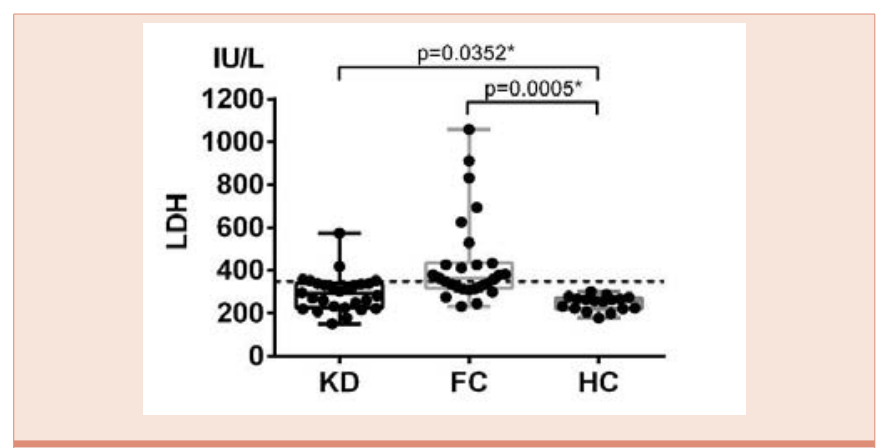

Figure 1: Serum LDH levels elevated significantly in KD and febrile non-KD patients. $\mathrm{LDH}$ levels were respectively measured in KD, compared with FC and $\mathrm{HC}$. Compared with $\mathrm{HC}$, sera LDH levels were significantly increase in $\mathrm{KD}(p=0.0352)$ and febrile controls $(p=0.0005)$, suggesting $\mathrm{LDH}$ not a specific marker for KD diagnosis. FC: fertile non-KD control.

Figure 2: Plasmid construction, protein expression and purification of LDHA. LDHA was over expressed according to standard procedures. LDHA gene was amplified by PCR and then analyzed by agarose gel electrophoresis in Panel A. Panel B showed a diagrammed process, including digestion, plasmid construction and transformation. Whole cell lysate protein from four recombinant E. Coli were respectively electrophoresed (Lane 2-5 in Panel C), compared with blank E. Coli control (Lane 1), blank plasmid control (Lane 7) and IPTG control (Lane 6). After ultrasonication, the majority of LDHA protein was detected in the precipitation (Lane 2 in Panel D), barely in the supernatant (Lane 1). Panel E showed purification of LDHA, followed by MS identification in Panel F.

( $p=0.038$, Figure 3C), with average OD of 0.663 vs 0.779 vs 0.769 . Serum anti-LDHA IgM antibodies were detected in none of KD or febrile non-KD patients.

Correlations of serum LDH levels and serum anti-LDHA IgA, IgG and IgM antibodies were analyzed respectively. If $\mathrm{p}$ value $<0.05$, statistical significance should be considered. As shown in Figure 3D$\mathrm{F}$, the results proved LDH levels not relating to serum IgA antibodies $\left(\mathrm{r}^{2}=0.02130, \mathrm{p}=0.4676\right)$, IgG antibodies $\left(\mathrm{r}^{2}=0.03887, \mathrm{p}=0.3243\right)$ and IgM antibodies $\left(\mathrm{r}^{2}=0.09043, \mathrm{p}=0.1275\right)$.

\section{Discussion}

LDH is extensively found in many human organs, which catalyzes the conversion of pyruvate to lactate or reverse, and converts $\mathrm{NAD}^{+}$ to NADH and back. Antigenicity of LDH isoenzymes hasn't been tested previously in $\mathrm{KD}$, though $\mathrm{LDH}$ level is often regarded as an auxiliary diagnosis marker judging resistance to IVIG treatment $[17,18]$. Elevation of LDH levels is probably a result of long-time fever and complications especially small- and medium-sized vessel 

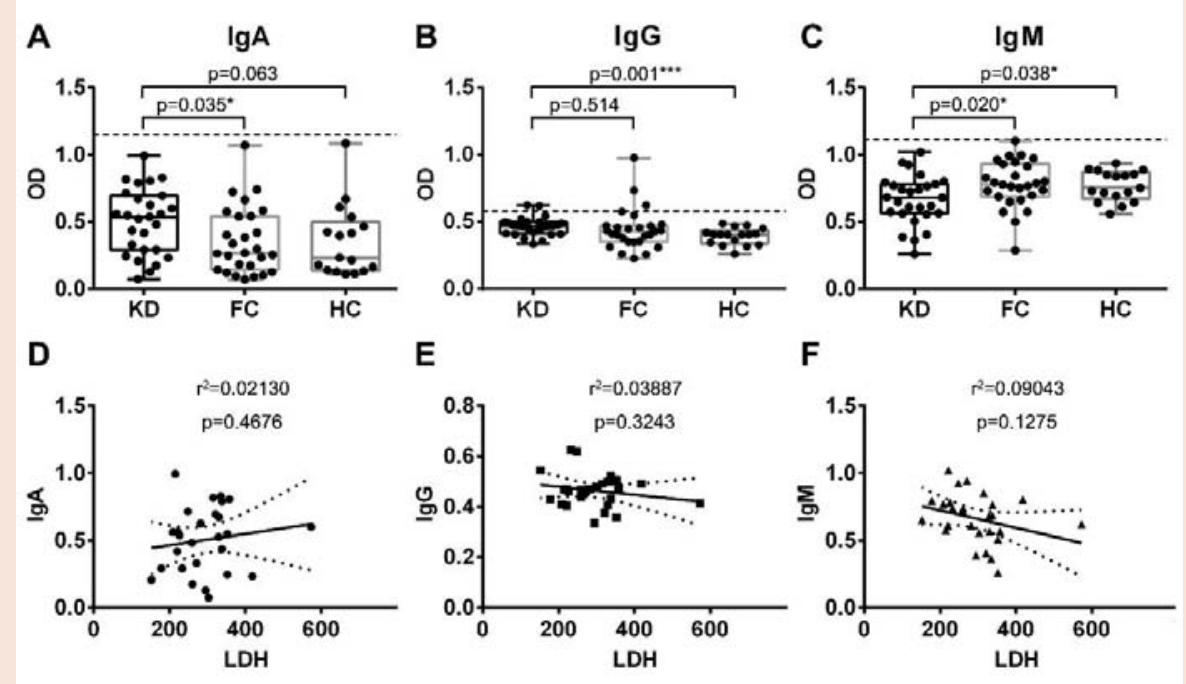

Figure 3: ELISA analysis of serum anti-LDHA IgA, IgG and IgM antibody levels. Panel A showed a significant elevation of serum anti-LDHA IgA levels in KD patients, compared with FC ( $p=0.035)$. For anti-LDHA IgG analysis in Panel $B$, an increase was also detected in KD compared with HC $(p=0.001)$. While a decline was observed in serum anti-LDHA IgM levels in Panel C, compared with FC $(p=0.020)$ and HC $(p=0.038)$. Panel D-F respectively showed correlation analysis between serum LDH levels and anti-LDHA IgA, IgG and IgM levels, and the results proved no obvious linear relationship.

vasculitis in KD. Since implicating with many organs in $\mathrm{KD}$, LDH is released from various parts of body. But the increasement of $\mathrm{LDH}$ is an aspecific marker, on account of a higher elevation finding in other febrile conditions including mycoplasma pneumonia, viral pneumonia, acute pneumonia, bronchopneumonia and multiple infection pneumonia.

Only IgA group showed observed elevation in $\mathrm{KD}$ compared with febrile control ( $p<0.05)$. For IgG group, even though a statistical significance was observed between KD and $\mathrm{HC}(\mathrm{p}=0.001)$, differences were not found between $\mathrm{KD}$ and febrile patients. None of IgA or IgM isotype of anti-LDHA antibodies were detected in all cases, and detection rate of serum anti-LDHA IgG antibodies was low in KD (7\%).

\section{Conclusion}

In conclusion, our results demonstrated the specific anti-LDH antibodies were not found in patients with KD or other similar febrile conditions, confirmed although $\mathrm{LDH}$ is associated to $\mathrm{KD}$, but does not play a autoimmune role in this condition.

\section{Acknowledgement}

This work was supported by National Natural Science Foundation of China (No. 81571592, 31371203), Beijing Nova Program of Science and Technology (No. 2007B024), and Program for New Century Excellent Talents in University (No. NECT-12-0773).

\section{References}

1. Kawasaki T (1967) Acute febrile mucocutaneous syndrome with lymphoid involvement with specific desquamation of the fingers and toes in children. Jpn J Allergy 16: 178-222.

2. Shulman ST, Rowley AH (1997) Etiology and pathogenesis of Kawasaki disease. Progress in Pediatric cardiology 6: 187-192.
3. Bayers S, Shulman ST, Paller AS (2013) Kawasaki disease: Part I. Diagnosis, clinical features, and pathogenesis. J Am Acad Dermatol 69: 501.

4. Principi N, Rigante D, Esposito S (2013) The role of infection in Kawasaki syndrome. J Infection 67: 1-10.

5. Eleftheriou D, Brogan $\mathrm{P}$ (2009) Vasculitis in children. Best Pract Res $\mathrm{Cl} R \mathrm{Rh}$ 23: 309-323.

6. Kuo HC, Yang KD, Chang WC, Ger LP, Hsieh KS (2012) Kawasaki disease: an update on diagnosis and treatment. Pediatr Neonatol 53: 4-11.

7. Sánchez-Manubens J, Bou R, Anton J (2014) Diagnosis and classification of Kawasaki disease. J Autoimmun 48-49: 113-117.

8. Kato H, Sugimura T, Akagi T, Sato N, Hashino K, et al. (1996) Long-term consequences of Kawasaki disease A 10-to 21-year follow-up study of 594 patients. Circulation 94: 1379-1385.

9. Hewins P, Jayne D (2010) Medium vessel vasculitis. Medicine 38: 93-96.

10. Falcini F, Capannini S, Rigante D (2011) Kawasaki syndrome: an intriguing disease with numerous unsolved dilemmas. Pediatr Rheumatol Online J 9: 17.

11. Bayers S, Shulman ST, Paller AS (2013) Kawasaki disease: Part II. Complications and treatment. J Am Acad Dermatol 69: 513.e1-8.

12. O Connell J, Sloand E (2013) Kawasaki Syndrome and Streptococcal Scarlet Fever: A Clinical Review. The Journal for Nurse Practitioners 9: 259-264.

13. Huang G, Ma X, Huang M, Chen S, Huang M, et al. (2013) Epidemiologic pictures of Kawasaki disease in Shanghai from 1998 through 2002. J Epidemiol 16: 9-14.

14. Morini F, Ronchetti MP, Bagolan $P$ (2009) Increased serum activity of lactate dehydrogenase in infants with necrotising enterocolitis: a potential marker of advanced disease. Paed Child Healt-CAN 19: S60-64.

15. Wan X, Wei L, Li H, Dong M, Lin Q, et al. (2013) High pretreatment serum lactate dehydrogenase level correlates with disease relapse and predicts an inferior outcome in locally advanced nasopharyngeal carcinoma. Eur J Cancer 49: 2356-2364.

16. Inamura N, Miyashita N, Hasegawa S, Kato A, Fukuda Y, et al. (2014) Management of refractory Mycoplasma pneumoniae pneumonia: Utility of measuring serum lactate dehydrogenase level. J Infect Chemother 20: 270273. 
17. Miyashita N, Obase Y, Ouchi K, Kawasaki K, Kawai Y, et al. (2007) Clinical features of severe Mycoplasma pneumoniae pneumonia in adults admitted to an intensive care unit. J Med Microbiol 56: 1625-1629.

18. Egami K, Muta H, Ishii M, Suda K, Sugahara Y, et al. (2006) Prediction of resistance to intravenous immunoglobulin treatment in patients with Kawasaki disease. J Pediatr 149: 237-240.

19. Fukunishi M, Kikkawa M, Hamana K, Onodera T, Matsuzaki K, et al. (2000) Prediction of non-responsiveness to intravenous high-dose $Y$-globulin therapy in patients with Kawasaki disease at onset. J Pediatr 137: 172-176.

20. Wang W, Gong F, Zhu W, Fu S, Zhang Q (2015) Macrophage activation syndrome in Kawasaki Disease: More common than we thought? Semin Arthritis Rheum 44: 405-410.

21. Muise A, Tallett SE, Silverman ED (2003) Are children with Kawasaki disease and prolonged fever at risk for macrophage activation syndrome? Pediatrics 112: e495-497.

22. Japanese Circulation Society Joint Research Group (2005) Guidelines for diagnosis and management of cardiovascular sequelae in Kawasaki disease. Pediatr Int 47: 711-732.
23. Duan C, Du ZD, Wang Y, Jia LQ (2014) Effect of pravastatin on endothelial dysfunction in children with medium to giant coronary aneurysms due to Kawasaki disease. World J Pediatr 10: 232-237.

24. Yang H, Du Z, Fu P (2013) Clinical features of recurrent Kawasaki disease and its risk factors. Eur J Pediatr 172: 1641-1647.

25. Du H, Chen G, Wang S, Wang S, Li S, et al. (2012) Immunological screening and characterization of highly specific monoclonal antibodies against $20 \mathrm{kDa}$ hGH. Bioanalysis 4: 2161-2168.

26. Xun Y, Chen P, Yan H, Yang W, Shi L, et al. (2014) Identification of prohibitin as an antigen in Behcet's disease. Biochem Bioph Res Co 451: 389-393.

27. Du H, Liu J, Xun Y, Liang J, Li S, et al. (2014) Determination of Deoxynivalenol, Zearalenone, Aflatoxin B1, and Ochratoxin by an EnzymeLinked Immunosorbent Assay. Anal Lett 47: 1912-1920.

28. Du HW, Chen GY, Yang CH, Li CB, Xun YP, et al. (2014) Multiple correlations of $\mathrm{mRNA}$ expression and protein abundance in human cytokine profile. Mole Bio Rep 41: 6985-6993. 